\title{
Thresholds in the Lattice of Subspaces of $\left(\mathbb{F}_{q}\right)^{n}$
}

\author{
Benjamin Rossman \\ University of Toronto
}

October 3, 2019

\begin{abstract}
Let $Q$ be an ideal (downward-closed set) in the lattice of linear subspaces of $\left(\mathbb{F}_{q}\right)^{n}$, ordered by inclusion. For $0 \leqslant k \leqslant n$, let $\mu_{k}(Q)$ denote the fraction of $k$-dimensional subspaces that belong to $Q$. We show that these densities satisfy

$$
\mu_{k}(Q)=\frac{1}{1+z} \quad \Longrightarrow \quad \mu_{k+1}(Q) \leqslant \frac{1}{1+q z} .
$$

This implies a sharp threshold theorem: if $\mu_{k}(Q) \leqslant 1-\varepsilon$, then $\mu_{\ell}(Q) \leqslant \varepsilon$ for $\ell=k+O\left(\log _{q}(1 / \varepsilon)\right)$.
\end{abstract}

\section{Introduction}

Let $\mathcal{L}_{q}(n)$ be the lattice of linear subspaces of $\left(\mathbb{F}_{q}\right)^{n}$, ordered by inclusion. Let $Q$ be a nontrivial ideal in $\mathcal{L}_{q}(n)$ (that is, a nonempty proper subset of $\mathcal{L}_{q}(n)$ such that $A \in Q$ implies $B \in Q$ for all $B \subset A)$. For $0 \leqslant k \leqslant n$, let $\mu_{k}(Q)$ denote the fraction of $k$-dimensional subspaces that belong to $Q$. Densities $\mu_{k}(Q)$ are known to be non-increasing: thus,

$$
1=\mu_{0}(Q) \geqslant \cdots \geqslant \mu_{t-1}(Q) \geqslant 1 / 2>\mu_{t}(Q) \geqslant \cdots \geqslant \mu_{n}(Q)=0
$$

for a unique $t$. This paper addresses the question: How quickly must $\mu_{k}(Q)$ transition from $1-o(1)$ to $o(1)$ ?

It follows from known results (described in 92 ) that

$$
\mu_{\lfloor(t-1) / c\rfloor}(Q) \geqslant 2^{-1 / c} \text { and } \quad \mu_{\lceil c t\rceil}(Q) \leqslant 2^{-c}
$$

for all $c \geqslant 1$. This is the $q$-analog of the Bollobás-Thomason Theorem [3], which speaks of ideals in the boolean lattice $\mathcal{P}(n)$ of subsets of $\{1, \ldots, n\}$.

On the one hand, $\mathcal{L}_{q}(n)$ is the $q$-analog of $\mathcal{P}(n)$; on the other hand, it is a sub-lattice of $\mathcal{P}\left(q^{n}\right)$. This raises the question: Do $k$-subspace densities of ideals in $\mathcal{L}_{q}(n)$ scale like $k$-subset densities in $\mathcal{P}(n)$ or like $q^{k}$-subset densities in $\mathcal{P}\left(q^{n}\right)$ ? Quantitatively, the latter suggests we should expect that

$$
\mu_{t-1-c}(Q) \geqslant 1-q^{-c} \text { and } \mu_{t+c}(Q) \leqslant q^{-c} .
$$

for all integers $c \geqslant 1$. This is precisely what we show.

Our main result actually concerns shadows in the subspace lattice. Let $\mathcal{L}_{q}(n, k)$ denote the set of $k$-dimensional subspaces of $\left(\mathbb{F}_{q}\right)^{n}$. For $1 \leqslant k \leqslant n$ and $S \subseteq \mathcal{L}_{q}(n, k)$, the shadow of $S$ is the set $\triangle S \subseteq \mathcal{L}_{q}(n, k-1)$ defined by $\triangle S:=\left\{B \in L_{n, k-1}: \exists A \in S, A \subset B\right\}$. We show: 
Theorem 1. For all $1 \leqslant k \leqslant n$ and $S \subseteq \mathcal{L}_{q}(n, k)$, if $\mu_{k}(S)=(1+z)^{-1}$ where $z \in \mathbb{R}_{\geqslant 0}$, then

$$
\mu_{k-1}(\triangle S) \geqslant\left(1+\frac{q\left(q^{k-1}-1\right)\left(q^{n-k}-1\right)}{\left(q^{k}-1\right)\left(q^{n-k+1}-1\right)} \cdot z\right)^{-1} \geqslant\left(1+\frac{z}{q}\right)^{-1} .
$$

The first inequality in Theorem 1 is tight in two cases:

- when $S$ is the set of $k$-dimensional subspaces of a fixed $n$-1-dimensional space $\left(z=\frac{q^{n}-q^{n-k}}{q^{n-k}-1}\right)$, as well as

- when $S$ is the set of $k$-dimensional subspaces not containing a fixed 1-dimensional space $\left(z=\frac{q^{k}-1}{q^{n}-q^{k}}\right)$.

For values of $z$ between $\frac{q^{k}-1}{q^{n}-q^{k}}$ and $\frac{q^{n}-q^{n-k}}{q^{n-k}-1}$, Theorem 2 improves the lower bound on $\mu_{k-1}(\triangle S)$ given by a $q$-analog of the Kruskal-Katona Theorem due to Chowdhury and Patkós [5].

A sharp threshold theorem for $\mathcal{L}_{q}(n)$ follows immediately from Theorem 1 and the observation that $\triangle\left(Q \cap \mathcal{L}_{q}(n, k)\right) \subseteq Q \cap \mathcal{L}_{q}(n, k-1)$ for ideals $Q$.

Theorem 2. For every ideal $Q$ in $\mathcal{L}_{q}(n)$ and $1 \leqslant k \leqslant n-1$, if $\mu_{k}(Q)=(1+z)^{-1}$, then $\mu_{k-1}(Q) \geqslant$ $(1+(z / q))^{-1}$ and $\mu_{k+1}(Q) \leqslant(1+q z)^{-1}$. As a consequence, if $\mu_{k}(Q) \leqslant 1-\varepsilon$, then $\mu_{\ell}(Q) \leqslant \varepsilon$ for $\ell=k+O\left(\log _{q}(1 / \varepsilon)\right)$.

The rest of the paper is organized as follows. In $\S 2$ we describe the previous $q$-analogs of the Kruskal-Katona and Bollobás-Thomason Theorems and their dual versions. In $₫ 3$ we prove Theorem 1 using well-known tools (the Expander Mixing Lemma and bounds on the eigenvalues of Grassmann graphs). In $\$ 4$ we discuss the tightness of the results. Finally, in $\$ 5$ we give an application of Theorem 2 to a problem in query complexity.

\section{2 q-Analogs of Kruskal-Katona and Bollobás-Thomason}

For $x \in \mathbb{R}_{\geqslant 0}$, let $[x]_{q}:=\frac{q^{x}-1}{q-1}$. The (Gaussian) $q$-binomial coefficient $\left[\begin{array}{l}x \\ k\end{array}\right]_{q}$ is defined by

$$
\left[\begin{array}{l}
x \\
k
\end{array}\right]_{q}:=\prod_{i=0}^{k-1} \frac{[x-i]_{q}}{[k-i]_{q}} .
$$

Note that $[0]_{q}=0$ and $[1]_{q}=1$ and $\left|\mathcal{L}_{q}(n, k)\right|=\left[\begin{array}{l}n \\ k\end{array}\right]_{q}=\left[\begin{array}{c}n \\ n-k\end{array}\right]_{q}$ for integers $n \geqslant k$.

Chowdhury and Patkós [5] proved a $q$-analog the Kruskal-Katona Theorem [8, 11], specifically a version due Keevash [10]. (See [14] for an alternative proof.)

Theorem 3 (q-Kruskal-Katona). For all $1 \leqslant k \leqslant n$ and $S \subseteq \mathcal{L}_{q}(n, k)$, if $|S|=\left[\begin{array}{l}x \\ k\end{array}\right]_{q}$, then $|\triangle S| \geqslant$ $\left[\begin{array}{c}x \\ k-1\end{array}\right]_{q}$. Moreover, this bound is tight when $S$ is the set of $k$-dimensional subspaces of a fixed $\ell$ dimensional space where $k \leqslant \ell \leqslant n$.

Note that the parameter $n$ (the dimension of the ambient vector space) plays no role in this bound, in contrast to Theorem 1, It turns out Theorem 3 is slack when $n-1<x<n$; this is precisely where Theorem 1 gives an improvement (as we discuss in $\$ 4$ ).

Combining Theorem 3 with the inequality $\left(\left[\begin{array}{c}x \\ k-1\end{array}\right]_{q} /\left[\begin{array}{c}n \\ k-1\end{array}\right]_{q}\right)^{k} \geqslant\left(\left[\begin{array}{l}x \\ k\end{array}\right]_{q} /\left[\begin{array}{c}n \\ k\end{array}\right]_{q}\right)^{k-1}$ for all $k \leqslant x \leqslant n$, we have the following $q$-analog of the Bollobás-Thomason Theorem [3] for the boolean lattice $\mathcal{P}(n)$. 
Theorem 4 (q-Bollobás-Thomason). For every ideal $Q$ in $\mathcal{L}_{q}(n)$,

$$
\mu_{1}(Q) \geqslant \mu_{2}(Q)^{1 / 2} \geqslant \mu_{3}(Q)^{1 / 3} \geqslant \cdots \geqslant \mu_{n}(Q)^{1 / n}
$$

In particular, if $\mu_{t-1}(Q) \geqslant 1 / 2>\mu_{t}(Q)$, then $\mu_{\lfloor(t-1) / c\rfloor}(Q) \geqslant 2^{-1 / c}$ and $\mu_{\lceil c t\rceil}(Q) \leqslant 2^{-c}$ for all $c \geqslant 1$.

If we regard $Q$ as a sequence of ideals in $\mathcal{L}_{q}(n)$, one for each $n$, then Theorem 4 implies that every nontrivial $Q$ has a threshold function $t(n)$, meaning that $\mu_{k(n)}(Q)=1-o(1)$ for all $k(n)=o(t(n))$ and $\mu_{\ell(n)}(Q)=o(1)$ for all $\ell(n)=\omega(t(n))$. In the boolean lattice $\mathcal{P}(n)$, nothing more can be said in general, although certain classes of ideals in $\mathcal{P}(n)$, such as monotone graph properties when $n=\left(\begin{array}{c}m \\ 2\end{array}\right)$, are known to have sharp thresholds such that $\mu_{k(n)}(Q)=1-o(1)$ and $\mu_{\ell(n)}(Q)=o(1)$ for some $k(n)=t(n)-o(t(n))$ and $\ell(n)=t(n)+o(t(n))$ (see [6]). In the same sense, Theorem 2 shows that every sequence of nontrivial ideals in $\mathcal{L}_{q}(n)$ has a sharp threshold.

\subsection{Dual versions of Theorems 3 and 4}

For a subspace $A$ of $\left(\mathbb{F}_{q}\right)^{n}$, the orthogonal complement is defined by

$$
A^{\perp}:=\left\{b \in\left(\mathbb{F}_{q}\right)^{n}: \sum_{i=1}^{n} a_{i} b_{i}=0 \text { for all } a \in A\right\} .
$$

Note that $\operatorname{dim}\left(A^{\perp}\right)=n-\operatorname{dim}(A)$ and $\left(A^{\perp}\right)^{\perp}=A$ and $B \subseteq A \Longrightarrow A^{\perp} \subseteq B^{\perp}$.

For every ideal $Q$ in $\mathcal{L}_{q}(n)$, there is a dual ideal $Q^{*}:=\left\{A \in \mathcal{L}_{q}(n): A^{\perp} \notin Q\right\}$ satisfying $\mu_{k}\left(Q^{*}\right)=1-\mu_{n-k}(Q)$. Applying Theorem 4 to $Q^{*}$ yields:

Theorem 5 (Dual q-Bollobás-Thomason). For every ideal $Q$ in $\mathcal{L}_{q}(n)$,

$$
1-\mu_{n-1}(Q) \geqslant\left(1-\mu_{n-2}(Q)\right)^{1 / 2} \geqslant\left(1-\mu_{n-3}(Q)\right)^{1 / 3} \geqslant \cdots \geqslant\left(1-\mu_{0}(Q)\right)^{1 / n} .
$$

In particular, $\mu_{\lfloor c(t-1)+(1-c) n\rfloor}(Q) \geqslant 1-2^{-c}$ and $\mu_{\lceil t / c+(1-1 / c) n\rceil}(Q) \leqslant 1-2^{-1 / c}$ for all $c \geqslant 1$. (This improves Theorem 4 when $t \geqslant n / 2$.)

Similarly, there is a dual version of Theorem 3. It may be helpful to include the proof, since we will use a similar argument in 93 ,

Theorem 6 (Dual $q$-Kruskal-Katona). For all $1 \leqslant k \leqslant n$ and $n-k+1 \leqslant y \leqslant n$ and $S \subseteq \mathcal{L}_{q}(n, k)$, if $|S|=\left[\begin{array}{l}n \\ k\end{array}\right]_{q}-\left[\begin{array}{c}y \\ n-k\end{array}\right]_{q}$, then $|\triangle S| \geqslant\left[\begin{array}{c}n \\ k-1\end{array}\right]_{q}-\left[\begin{array}{c}y \\ n-k+1\end{array}\right]_{q}$.

Proof. We will assume $|\triangle S|<\left[\begin{array}{c}n \\ k-1\end{array}\right]_{q}-\left[\begin{array}{c}y \\ n-k+1\end{array}\right]_{q}$ and prove that $|S|<\left[\begin{array}{l}n \\ k\end{array}\right]_{q}-\left[\begin{array}{c}y \\ n-k\end{array}\right]_{q}$. Define $T \subseteq \mathcal{L}_{q}(n, n-k+1)$ by

$$
T:=\left\{B^{\perp}: B \in \mathcal{L}_{q}(n, k-1) \backslash \triangle S\right\} .
$$

Note that $|T|=\left[\begin{array}{c}n \\ k-1\end{array}\right]_{q}-|\triangle S|=\left[\begin{array}{c}y \\ n-k+1\end{array}\right]_{q}$. Therefore, Theorem 3 implies $|\triangle T|>\left[\begin{array}{c}y \\ n-k\end{array}\right]_{q}$.

For all $A \in \mathcal{L}_{q}(n, k)$, observe that

$$
\begin{aligned}
A^{\perp} \in \triangle T & \Longleftrightarrow \exists B \in \mathcal{L}_{q}(k-1) \backslash \triangle S, A^{\perp} \subset B^{\perp} \\
& \Longleftrightarrow \exists B \in \mathcal{L}_{q}(k-1) \backslash \triangle S, B \subset A \\
& \Longrightarrow A \notin S .
\end{aligned}
$$

Therefore, $S \subseteq\left\{A \in \mathcal{L}_{q}(n, k): A^{\perp} \notin \triangle T\right\}$. We conclude that $|S|=\left[\begin{array}{l}n \\ k\end{array}\right]_{q}-|\triangle T|<\left[\begin{array}{l}n \\ k\end{array}\right]_{q}-\left[\begin{array}{c}y \\ n-k\end{array}\right]_{q}$, as required. 


\section{Proof of Theorem 1}

The proof of Theorem 1 involves bounding the edge-expansion of sets in the Grassmann graph $J_{q}(n, k)$. We state the required definitions and lemmas below. (See [12] for a much deeper study of expansion of Grassman graphs.)

Definition 7. For a $d$-regular graph $G=(V, E)$ and $S \subseteq V$, the edge-expansion of $S$ is defined by

$$
\Phi_{G}(S):=\frac{|E(S, \bar{S})|}{d|S|}
$$

where $E(S, \bar{S})$ is the set of edges between $S$ and $\bar{S}=V \backslash S$.

Lemma 8 (Expander Mixing Lemma [1]). Let $G=(V, E)$ be a d-regular graph and suppose the second largest eigenvalue (in absolute value) of the adjacency matrix of $G$ is at most $\lambda$. Then for all $S \subseteq V$,

$$
\left(1-\frac{\lambda}{d}\right)\left(1-\frac{|S|}{|V|}\right) \leqslant \Phi_{G}(S) \leqslant\left(1+\frac{\lambda}{d}\right)\left(1-\frac{|S|}{|V|}\right) .
$$

Definition 9. For $1 \leqslant k \leqslant n$, the Grassmann graph $J_{q}(n, k)$ is the $q[k]_{q}[n-k]_{q}$-regular graph with vertex set $\mathcal{L}_{q}(n, k)$ and edge set

$$
E_{J_{q}(n, k)}:=\left\{\left(A_{1}, A_{2}\right) \in \mathcal{L}_{q}(n, k) \times \mathcal{L}_{q}(n, k): \operatorname{dim}\left(A_{1} \cap A_{2}\right)=k-1\right\} .
$$

Lemma 10 (Spectrum of $J_{q}(n, k)[4]$ ). The adjacency matrix of $J_{q}(n, k)$ has eigenvalue $q^{i+1}[k-$ $i]_{q}[n-k-i]_{q}-[i]_{q}$ with multiplicity $\left[\begin{array}{c}n \\ i\end{array}\right]_{q}-\left[\begin{array}{c}n \\ i-1\end{array}\right]_{q}$ for each $0 \leqslant i \leqslant \min (k, n-k)$. In particular, the second largest eigenvalue (in absolute value) equals 1 if $k \in\{1, n-1\}$ and equals $q^{2}[k-1]_{q}[n-k-$ $1]_{q}-1$ if $2 \leqslant k \leqslant n-2$.

Lemmas 8 and 10 give the following lower bound on $\Phi_{J_{q}(n, k)}(S)$.

Lemma 11. For all $2 \leqslant k \leqslant n-2$ and $S \subseteq \mathcal{L}_{q}(n, k)$,

$$
\Phi_{J_{q}(n, k)}(S) \geqslant \frac{[n]_{q}}{q[k]_{q}[n-k]_{q}}\left(1-\mu_{k}(S)\right) .
$$

Proof. Lemma 8 implies the lower bound

$$
\Phi_{J_{q}(n, k)}(S) \geqslant\left(1-\frac{q^{2}[k-1]_{q}[n-k-1]_{q}-1}{[k]_{q}[n-k]_{q}}\right)\left(1-\mu_{k}(S)\right) .
$$

By a straightforward calculation,

$$
1-\frac{q^{2}[k-1]_{q}[n-k-1]_{q}-1}{q[k]_{q}[n-k]_{q}}=\frac{q^{n+1}-q^{n}-q+1}{q^{n+1}-q^{k+1}-q^{n-k+1}+q}=\frac{[n]_{q}}{q[k]_{q}[n-k]_{q}} .
$$

We next show an upper bound on $\Phi_{J_{q}(n, k)}(S)$ in terms of the ratio $\mu_{k}(S) / \mu_{k-1}(\triangle S)$.

Lemma 12. For all $1 \leqslant k \leqslant n$ and $\emptyset \subset S \subseteq \mathcal{L}_{q}(n, k)$,

$$
\Phi_{J_{q}(n, k)}(S) \leqslant \frac{[n-k+1]_{q}}{q[n-k]_{q}}\left(1-\frac{\mu_{k}(S)}{\mu_{k-1}(\triangle S)}\right) .
$$


Proof. For $B \in \triangle S$, let $S_{B}:=\{A \in S: B \subset A\}$. We have $\sum_{B \in \triangle S}\left|S_{B}\right|=[k]_{q}|S|$ and, by the Cauchy-Schwarz inequality,

$$
\sum_{B \in \triangle S}\left|S_{B}\right|^{2} \geqslant \frac{\left(\sum_{B \in \triangle S}\left|S_{B}\right|\right)^{2}}{|\triangle S|}=\frac{\left([k]_{q}|S|\right)^{2}}{|\triangle S|}
$$

Therefore,

$$
\begin{aligned}
\left|E_{J_{q}(n, k)}(S, \bar{S})\right|=\sum_{B \in \triangle S}\left|S_{B} \times \bar{S}_{B}\right| & =\sum_{B \in \triangle S}\left|S_{B}\right|\left([n-k+1]_{q}-\left|S_{B}\right|\right) \\
& \leqslant[k]_{q}|S|\left([n-k+1]_{q}-\frac{[k]_{q}|S|}{|\triangle S|}\right) .
\end{aligned}
$$

We now have

$$
\Phi_{J_{q}(n, k)}(S)=\frac{\left|E_{J_{q}(n, k)}(S, \bar{S})\right|}{q[k]_{q}[n-k]_{q}|S|} \leqslant \frac{[n-k+1]_{q}}{q[n-k]_{q}}-\frac{[k]_{q}}{q[n-k]_{q}} \cdot \frac{|S|}{|\triangle S|} .
$$

The lemma now follows from the equality

$$
\frac{[k]_{q}}{q[n-k]_{q}} \cdot \frac{|S|}{|\triangle S|}=\frac{[k]_{q}\left[\begin{array}{l}
n \\
k
\end{array}\right]_{q}}{q[n-k]_{q}\left[\begin{array}{c}
n \\
k-1
\end{array}\right]_{q}} \cdot \frac{\mu_{k}(S)}{\mu_{k-1}(\triangle S)}=\frac{[n-k+1]_{q}}{q[n-k]_{q}} \cdot \frac{\mu_{k}(S)}{\mu_{k-1}(\triangle S)} .
$$

We are ready to prove:

Theorem 1 (restated). For all $1 \leqslant k \leqslant n$ and $S \subseteq \mathcal{L}_{q}(n, k)$, if $\mu_{k}(S)=(1+z)^{-1}$ where $z \in \mathbb{R}_{\geqslant 0}$, then

$$
\mu_{k-1}(\triangle S) \geqslant\left(1+\frac{q\left(q^{k-1}-1\right)\left(q^{n-k}-1\right)}{\left(q^{k}-1\right)\left(q^{n-k+1}-1\right)} \cdot z\right)^{-1} \geqslant\left(1+\frac{z}{q}\right)^{-1} .
$$

Proof. The second inequality is by a straightforward calculation:

$$
\frac{q\left(q^{k-1}-1\right)\left(q^{n-k}-1\right)}{\left(q^{k}-1\right)\left(q^{n-k+1}-1\right)}=\frac{1}{q}\left(1-\frac{(q-1)\left(q^{n-k+1}+q^{k}-q-1\right)}{\left(q^{k}-1\right)\left(q^{n-k+1}-1\right)}\right) \leqslant \frac{1}{q} .
$$

For the first inequality, consider the case that $k \in\{1, n\}$. In both cases, we have $\mu_{k-1}(\triangle S)=1$ for every nonempty $S \subseteq \mathcal{L}_{q}(n, k)$. Therefore, the inequality holds (moreover, with equality since $\left.[k-1]_{q}[n-k]_{q}=0\right)$.

Next, consider the case that $2 \leqslant k \leqslant n-2$. In this case, Lemmas 11 and 12 imply

$$
\frac{[n]_{q}}{q[k]_{q}[n-k]_{q}}\left(1-\mu_{k}(S)\right) \leqslant \Phi_{J_{q}(n, k)}(S) \leqslant \frac{[n-k+1]_{q}}{q[n-k]_{q}}\left(1-\frac{\mu_{k}(S)}{\mu_{k-1}(\triangle S)}\right) .
$$

Therefore,

$$
\frac{[n]_{q}}{[k]_{q}[n-k+1]_{q}}\left(1-\mu_{k}(S)\right) \leqslant 1-\frac{\mu_{k}(S)}{\mu_{k-1}(\triangle S)} .
$$


Substituting $(1+z)^{-1}$ for $\mu_{k}(S)$, this rearranges to

$$
\mu_{k-1}(\triangle S) \geqslant\left(1+\left(1-\frac{[n]_{q}}{[k]_{q}[n-k+1]_{q}}\right) z\right)^{-1}=\left(1+\frac{q\left(q^{k-1}-1\right)\left(q^{n-k}-1\right)}{\left(q^{k}-1\right)\left(q^{n-k+1}-1\right)} \cdot z\right)^{-1} .
$$

We derive the remaining case $k=n-1$ from the case $k=2$ via duality. Letting $S \subseteq \mathcal{L}_{q}(n, n-1)$, we will assume that

$$
\mu_{n-2}(S)<\left(1+\frac{q\left(q^{n-2}-1\right)(q-1)}{\left(q^{2}-1\right)\left(q^{n-1}-1\right)} \cdot z\right)^{-1}
$$

and show that $\mu_{n-1}(S)<(1+z)^{-1}$. Let $T:=\left\{B^{\perp}: B \in \mathcal{L}_{q}(n, n-2) \backslash \triangle S\right\}$ and note that

$$
\mu_{2}(T)=1-\mu_{n-2}(\triangle S)>1-\left(1+\frac{q\left(q^{n-2}-1\right)(q-1)}{\left(q^{2}-1\right)\left(q^{n-1}-1\right)} \cdot z\right)^{-1}=\left(1+\frac{\left(q^{2}-1\right)\left(q^{n-1}-1\right)}{z \cdot q\left(q^{n-2}-1\right)(q-1)}\right)^{-1} .
$$

From the case $k=2$, we have

$$
\mu_{1}(\triangle T) \geqslant\left(1+\frac{q\left(q^{n-2}-1\right)(q-1)}{\left(q^{2}-1\right)\left(q^{n-1}-1\right)} \cdot\left(\mu_{2}(T)^{-1}-1\right)\right)^{-1}>\left(1+\frac{1}{z}\right)^{-1} .
$$

Since $S \subseteq\left\{A \in \mathcal{L}_{q}(n, n-1): A^{\perp} \notin \triangle T\right\}$ (as in the proof of Theorem [6), it follows that

$$
\mu_{n-1}(S) \leqslant 1-\mu_{1}(\triangle T)<1-\left(1+\frac{1}{z}\right)^{-1}=(1+z)^{-1},
$$

as required.

We remark that Theorem 1 is self-dual: for any $1 \leqslant k \leqslant n$ and $S \subseteq \mathcal{L}_{q}(n, k)$, we get the same inequality between $\mu_{k}(S)$ and $\mu_{k-1}(\triangle S)$ as between $1-\mu_{n-k}(\triangle T)$ and $1-\mu_{n-k+1}(T)$ where $T:=\left\{B^{\perp}: B \in \mathcal{L}_{q}(n, k-1) \backslash \triangle S\right\}$.

\section{Tightness of the result}

Fix a flag $V_{0} \subset V_{1} \subset \cdots \subset V_{n}=\left(\mathbb{F}_{q}\right)^{n}$. (Without loss of generality, we may take $V_{k}=\left\{u \in\left(\mathbb{F}_{q}\right)^{n}\right.$ : $\left.u_{k+1}=\cdots=u_{n}=0\right\}$.) For $1 \leqslant j \leqslant n$, let $Q_{\widehat{j}}$ be the ideal

$$
Q_{\widehat{j}}:=\left\{A \in \mathcal{L}_{q}(n): A \cap\left(V_{j}-V_{j-1}\right)=\emptyset\right\} .
$$

In particular, $Q_{\widehat{1}}$ is the set of subspaces of $\left(\mathbb{F}_{q}\right)^{n}$ that do not contain $V_{1}$, while $Q_{\widehat{n}}$ is the set of subspaces contained in $V_{n-1}$. It can be shown (by some tedious calculations) that

(i) $\mu_{n-j}\left(Q_{\widehat{j}}\right)>1 / 2>\mu_{n-j+1}\left(Q_{\widehat{j}}\right)$,

(ii) if $2 \leqslant k \leqslant n-1$ and $\mu_{k}\left(Q_{\widehat{j}}\right)=(1+z)^{-1}$, then $\left(1+\frac{z}{q^{2}}\right)^{-1} \geqslant \mu_{k-1}\left(Q_{\widehat{j}}\right) \geqslant\left(1+\frac{z}{q}\right)^{-1}$,

(iii) $\mu_{k}\left(Q_{\widehat{n}}\right)=\frac{\left[\begin{array}{c}n-1 \\ k\end{array}\right]_{q}}{\left[\begin{array}{l}n \\ k\end{array}\right]_{q}}=\frac{[n-k]_{q}}{[n]_{q}}=\left(1+\frac{q^{n-k}\left(q^{k}-1\right)}{\left(q^{n-k}-1\right)}\right)^{-1}$, 
(iv) $\mu_{k}\left(Q_{\widehat{1}}\right)=1-\frac{\left[\begin{array}{l}n-1 \\ k-1\end{array}\right]_{q}}{\left[\begin{array}{l}n \\ k\end{array}\right]_{q}}=1-\frac{[k]_{q}}{[n]_{q}}=\left(1+\frac{\left(q^{k}-1\right)}{q^{k}\left(q^{n-k}-1\right)}\right)^{-1}$.

Inequalities (i) and (ii) show that Theorem 2 is essentially tight, no matter where in $\{1, \ldots, n\}$ the threshold for $Q$ occurs. Equations (iii) and (iv) show that the first inequality of Theorem 1 is tight

- when $S$ is the set of $k$-dimensional subspaces of a fixed $n$-1-dimensional space, as well as

- when $S$ is the set of $k$-dimensional subspaces not containing a fixed 1-dimensional space.

The first example is also tight for $q$-Kruskal-Katona (Theorem 3), while the second example is tight for the Dual $q$-Kruskal-Katona (Theorem 6). Taking the maximum of the bounds given by Theorem 1, 3] and 6, we get:

Corollary 13. For all $1 \leqslant k \leqslant n$ and $\emptyset \subset S \subset \mathcal{L}_{q}(n, k)$,

$|\triangle S| \geqslant \begin{cases}{\left[\begin{array}{c}x \\ k-1\end{array}\right]_{q}} & \text { if }|S|=\left[\begin{array}{l}x \\ k\end{array}\right]_{q} \text { where } k \leqslant x \leqslant n-1, \\ {\left[\begin{array}{c}n \\ k-1\end{array}\right]_{q}\left(1+\frac{z \cdot\left(q^{k-1}-1\right)}{q^{k-1}\left(q^{n-k+1}-1\right)}\right)^{-1}} & \text { if }|S|=\left[\begin{array}{l}n \\ k\end{array}\right]_{q}\left(1+\frac{z \cdot\left(q^{k}-1\right)}{q^{k}\left(q^{n-k}-1\right)}\right)^{-1} \text { where } 1 \leqslant z \leqslant q^{n}, \\ {\left[\begin{array}{c}n \\ k-1\end{array}\right]_{q}-\left[\begin{array}{c}y \\ n-k+1\end{array}\right]_{q}} & \text { if }|S|=\left[\begin{array}{l}n \\ k\end{array}\right]_{q}-\left[\begin{array}{c}y \\ n-k\end{array}\right]_{q} \text { where } n-k+1 \leqslant y \leqslant n-1 .\end{cases}$

Corollary 13 is known to be tight when $x$ or $y$ are integers (or $z \in\left\{1, q^{n}\right\}$, coinciding with cases $y=n-1$ and $x=n-1)$. In other cases, determining the optimal lower bound for $|\triangle S|$ in terms of $|S|$ remains an open problem. In contrast, note that the original Kruskal-Katona Theorem [8, 11] completely solves the shadow minimization problem in the boolean lattice: if $S$ is a family of $k$-element sets and $|S|=\left(\begin{array}{c}n_{k} \\ k\end{array}\right)+\left(\begin{array}{c}n_{k-1} \\ k-1\end{array}\right)+\cdots+\left(\begin{array}{c}n_{j} \\ j\end{array}\right)$ where $n_{k}>n_{k-1}>\cdots>n_{j}=j \geqslant 1$, then $|\triangle S| \geqslant\left(\begin{array}{c}n_{k} \\ k-1\end{array}\right)+\left(\begin{array}{c}n_{k-1} \\ k-2\end{array}\right)+\cdots+\left(\begin{array}{c}n_{j} \\ j-1\end{array}\right)$ and this bound is tight. Moreover, a family of nested solutions is given by the subsets of $\{1, \ldots, n\}$ in co-lexicographic order. The situation in $\mathcal{L}_{q}(n)$ appears more complicated, as nested solutions to the shadow minimization problem in $\mathcal{L}_{q}(n)$ are known not to exist [2, 7, 13].

\section{Application to a query problem}

We conclude by giving an application of Theorem 2 to a problem in query complexity. In this problem, $A$ is a hidden nontrivial subspace of $\left(\mathbb{F}_{2}\right)^{n}$ and the goal is to learn a nonzero element of $A$ with probability $\geqslant 1 / 2$ by making $m$ simultaneous (non-adaptive) monotone queries. What is the minimum $m$ for which this is possible? An upper bound of $O\left(n^{2}\right)$ is well-known (see [9]). The following theorem gives a matching lower bound of $\Omega\left(n^{2}\right)$.

Theorem 14. Let $\left(Q_{1}, \ldots, Q_{m}\right)$ be a joint distribution over ideals in the subspace lattice of $\left(\mathbb{F}_{2}\right)^{n}$ and let $f$ be a function $\{0,1\}^{m} \rightarrow\left(\mathbb{F}_{2}\right)^{n} \backslash\{\overrightarrow{0}\}$. Suppose that for every nontrivial subspace $A$ of $\left(\mathbb{F}_{2}\right)^{n}$, it holds that

$$
\underset{Q_{1}, \ldots, Q_{m}}{\operatorname{Pr}_{\left\{A \in Q_{1}\right\}}, \ldots, 1_{\left\{A \in Q_{m}\right\}}}\left[f\left(1_{1 / 2}\right] \geqslant 1 / 2\right.
$$

where $1_{\left\{A \in Q_{i}\right\}}$ is the indicator function for the event that $A \in Q_{i}$. Then $m=\Omega\left(n^{2}\right)$. 
This lower bound follows from combining Theorem [2 with some lemmas from the paper [9], which proves the special case of Theorem 14 where ideals $Q_{i}$ are restricted to be of the form $\left\{A \in L_{2}(n): A \cap U_{i}=\emptyset\right\}$ for subsets $U_{i} \subseteq\left(\mathbb{F}_{2}\right)^{n}$.

\section{References}

[1] Noga Alon and Fan RK Chung. Explicit construction of linear sized tolerant networks. Discrete Mathematics, 72(1-3):15-19, 1988.

[2] Sergei Bezrukov and Aart Blokhuis. A Kruskal-Katona type theorem for the linear lattice. European Journal of Combinatorics, 20(2):123-130, 1999.

[3] Béla Bollobás and Arthur G Thomason. Threshold functions. Combinatorica, 7(1):35-38, 1987.

[4] Andries E Brouwer and Willem H Haemers. Distance-regular graphs. In Spectra of Graphs, pages 177-185. Springer, 2012.

[5] Ameera Chowdhury and Balázs Patkós. Shadows and intersections in vector spaces. Journal of Combinatorial Theory, Series A, 117(8):1095-1106, 2010.

[6] Ehud Friedgut and Gil Kalai. Every monotone graph property has a sharp threshold. Proceedings of the American mathematical Society, 124(10):2993-3002, 1996.

[7] LH Harper and F Hergert. The isoperimetric problem in finite projective planes. Congressus Numerantium, pages 225-232, 1994.

[8] Gyula Katona. A theorem of finite sets. In Classic Papers in Combinatorics, pages 381-401. Springer, 2009.

[9] Akinori Kawachi, Benjamin Rossman, and Osamu Watanabe. The query complexity of witness finding. Theory of Computing Systems, 61(2):305-321, 2017.

[10] Peter Keevash. Shadows and intersections: stability and new proofs. Advances in Mathematics, 218(5):1685-1703, 2008.

[11] Joseph B Kruskal. The number of simplices in a complex. Mathematical optimization techniques, 10:251-278, 1963.

[12] Khot Subhash, Dor Minzer, and Muli Safra. Pseudorandom sets in Grassmann graph have near-perfect expansion. In 59th Annual IEEE Symposium on Foundations of Computer Science (FOCS), pages 592-601, 2018.

[13] Patricia K Ure. A study of $(0, n, n+1)$-sets and other solutions of the isoperimetric problem in finite projective planes. PhD thesis, California Institute of Technology, 1996.

[14] Jun Wang. Intersecting antichains and shadows in linear lattices. Journal of Combinatorial Theory, Series A, 118(7):2092-2101, 2011. 